\title{
EXPLORING UNIVERSALITY OF FEW-BODY PHYSICS BASED ON ULTRACOLD ATOMS NEAR FESHBACH RESONANCES
}

\author{
Nathan Gemelke, Chen-Lung Hung, Xibo Zhang and Cheng Chin* \\ James Franck Institute and Physics Department, University of Chicago, \\ Chicago, IL 60637, USA \\ *E-mail: cchin@uchicago.edu \\ Website: http://ultracold.uchicago.edu
}

\begin{abstract}
A universal characterization of interactions in few- and many-body quantum systems is often possible without detailed description of the interaction potential, and has become a defacto assumption for cold atom research. Universality in this context is defined as the validity to fully characterize the system in terms of two-body scattering length. We discuss universality in the following three contexts: closed-channel dominated Feshbach resonance, Efimov physics near Feshbach resonances, and corrections to the mean field energy of BoseEinstein condensates with large scattering lengths. Novel experimental tools and strategies are discussed to study universality in ultracold atomic gases: dynamic control of interactions, run-away evaporative cooling in optical traps, and preparation of few-body systems in optical lattices.
\end{abstract}

Keywords: Universality, Bose-Einstein condensation, Feshbach, Efimov, meanfield interaction

\section{Introduction}

Quantum gases of ultracold atoms distinguish themselves from other quantum systems in two unique and useful ways. First of all, the diluteness of the gases permits a very simple and accurate description of the effect of interactions. Degenerate gases of atoms can be described well by textbook models of fundamental and general interest. Extending beyond these, complexity can be built in slowly to study novel quantum phases, and even intractable mathematical models. This aspect has inspired new research in the vein of quantum simulation, promising far-reaching impact on the understanding of other quantum systems in nature, including condensed and nuclear matter.

A second gainful aspect of ultracold atomic gases lies in the ability 
to tune interactions via Feshbach resonant scattering. Exploitation of this feature, first observed in $1998,{ }^{1}$ has only fully matured in recent years, and promises numerous future applications. Full control of interaction in a quantum gas not only allows for an easy exploration of the quantum system in different interaction regimes, but also leads to new methods to observe dynamic evolution and to scrutinize quantum states in previously unimaginable ways. For example, projecting a complex many-body state onto a non-interacting single particle basis can be realized by diabatically switching off atomic interactions.

The majority of quantum gas systems studied to date admit a universal description of the effect of interaction. In this paper, we describe our approach to explore situations in which universality requires nontrivial extensions. Our experimental platform, based on optically trapped cesium atoms, exploits both of the aforementioned features, allowing us to address long-standing questions concerning the universality of an interacting gas. In particular, we will focus on three topics: universality and its minimal extensions in the study of dimer molecules, three-body Efimov states near Feshbach resonance, and beyond mean-field interactions in Bose-Einstein condensates. Finally, we will outline our approach to study few-body interactions and our experimental progress.

\section{Universality in $N$-body physics}

The connection of quantum degenerate atomic gases to other physical systems is made possible by the expected universality of physics at low temperatures. Here, universality arises when the quantum system is fully described by a single parameter, the two-body scattering length $a .^{2}$ Universality is well established in two-body, low energy scattering theory, where the $s$-wave scattering phase shift is $\eta=-\tan ^{-1} k a$, with $k$ the scattering wave number. In the many-body regime, universal behavior of dilute Bose-Einstein condensates (BECs) of different bosonic atomic species is expected for small and positive scattering lengths. Universality is further expected and verified in two-component degenerate Fermi gases with large scattering lengths, as in the BEC-BCS (Bardeen-Cooper-Schrieffer superfluid) crossover regime. ${ }^{3-5}$

Non-universal parameters, however, can play an important role in certain low energy few- and many-body systems, and represent the entrance of a richer underlying scattering physics. For example, binding energies of Efimov states in three-body systems ${ }^{6}$ and three-body interactions in BECs with large scattering lengths ${ }^{20}$ are expected to be non-universal. Both cases 
strongly depend on the three-body scattering phase shifts, which likely cannot be universally derived from $a .^{2}$

\section{Feshbach resonances}

\subsection{Origin of Feshbach resonance}

In cold atom experiments, Feshbach resonances occur when two free atoms interact in the scattering channel and resonantly couple to a bound molecular state in a closed channel. ${ }^{8}$ In many cases, the bound state can have a different magnetic moment than that of the scattering atoms, and resonant coupling between the channels can be induced by tuning the bound state energy with an external magnetic field.

Near a Feshbach resonance, the scattering phase shift $\eta$ follows the BreitWigner formula: ${ }^{9}$

$$
\eta=\eta_{\mathrm{bg}}-\tan ^{-1} \frac{\Gamma / 2}{E-E_{c}-\delta E},
$$

where $\eta_{\mathrm{bg}}$ is the background, or off-resonant, phase shift, $E=\hbar^{2} k^{2} / m$ is the scattering energy, $k$ is the scattering wave number, $m$ is twice the reduced mass, $\Gamma \propto k$ is the coupling strength between the scattering and bound states, $E_{c}$ is the energy of the bare bound state, and $\delta E$ is the self-energy shift.

At low scattering energies $E \rightarrow 0$, the (background) scattering length is given by $a_{(\mathrm{bg})}=-\tan \eta_{(\mathrm{bg})} / k \cdot{ }^{9}$ We further assume a linear Zeeman shift to the bound state $E_{c}=\delta \mu\left(B-B_{c}\right)$, where $\delta \mu$ is the relative magnetic moment between open and closed channels, and the bound state is shifted to the continuum when $B=B_{c}$. These allow us to derive scattering length in the standard resonance form $a=a_{\mathrm{bg}}\left[1-\Delta /\left(B-B_{0}\right)\right]$. Here $\Delta=\lim _{k \rightarrow 0} \Gamma /\left(2 k a_{\mathrm{bg}} \delta \mu\right)$ is the resonance width, $B_{0}=B_{c}-\delta E / \delta \mu$ is the resonance position. Note that $a$ diverges when $B=B_{0}$, or equivalently, $\eta=\left(N+\frac{1}{2}\right) \pi$, where $N$ is the number of molecular states below the continuum.

It is important to note that Feshbach resonance occurs not exactly when the bare state is tuned to the continuum $B=B_{c}$. From the van der Waals potential model, the resonance position offset is ${ }^{10}$

$$
B_{0}-B_{c}=-\frac{\delta E}{\delta \mu}=-\frac{r^{2}-r}{r^{2}-2 r+2} \Delta
$$


where $r=a_{\mathrm{bg}} / \bar{a}$ and $\bar{a}$ is the mean scattering length of the van der Waals potential. ${ }^{11}$

Eq. 2 shows that the difference in magnetic field between the bare state crossing and the resonance position $B_{0}-B_{c}$ is on the order of the resonance width $\Delta$ when $\left|a_{\mathrm{bg}}\right|>\bar{a}$.

\subsection{Non-universality of Feshbach molecules}

The two-channel nature of the interaction potential described in the previous section implies that, in the absence of Feshbach coupling, the scattering length of atoms in the entrance channel does not reveal the properties of the closed channel bound state. Thus, the properties of the molecular state are clearly non-universal. This point can also been seen in Fig. 1. When the molecular state is well below the continuum, the molecular energy approaches the bare state value $E_{c}=\delta \mu\left(B-B_{c}\right)$, which cannot be universally derived from mere knowledge of $a$.

When the magnetic field is tuned sufficiently near the Feshbach resonance, Feshbach coupling strongly modifies the nature of the bound state, whose character is now dominated by the open channel. In this regime, the molecular state does develop a universal behavior with a binding energy of $E_{b}=\hbar^{2} / m a^{2}$. The Universal regime can be seen in Fig. 1 (b) inset.

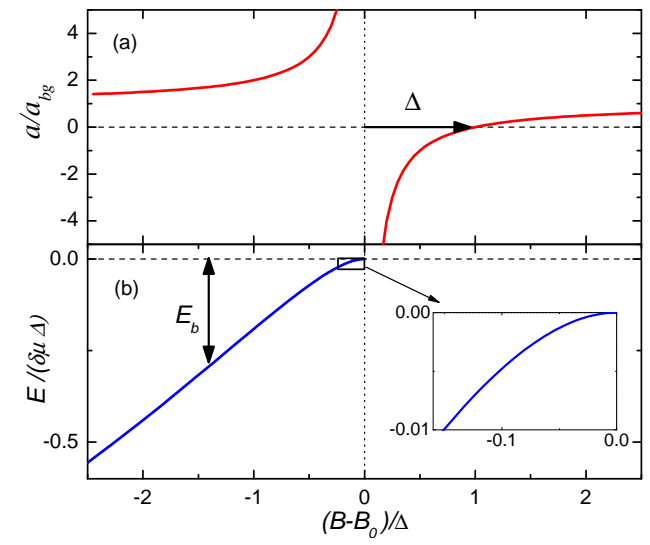

Fig. 1. Scattering length $a$ in (a) and molecular state energy $E$ in (b) near a magnetically tuned Feshbach resonance. $E_{b}>0$ is the binding energy. The inset shows the universal regime where $E_{b}=\hbar^{2} / m a^{2}$.

The transition between the non-universal and universal regimes differs 
for different resonances. One instructive way to see the transition behavior is to expand the molecular binding energy near the resonance, which, from a simplified two-channel model potential, ${ }^{12}$ gives

$$
E_{b} \approx \frac{\hbar^{2}}{m\left(a-\bar{a}-R^{*}\right)^{2}} .
$$

Here $\bar{a}, R^{*}=a_{\mathrm{bg}} E_{\mathrm{bg}} /(\delta \mu \Delta)$ and $E_{\mathrm{bg}}=\hbar^{2} / m a_{\mathrm{bg}}^{2},{ }^{13}$ are two leading order non-universal length scales associated with the finite interaction range and the coupling strength to the closed channel bound state, respectively. Universality is valid only when $a \gg \bar{a}$ and $R^{*}$. In particular, $R^{*}$, can be uncharacteristically large, $R^{*} \gg \bar{a}$, for narrow resonances . Resonances of this type are deemed closed-channel dominated and can have strong nonuniversal behavior.

\section{Efimov physics and Efimov states}

Efimov states are a set of three-body, long-range bound states which emerge when the pairwise interactions in a three-particle system are resonantly enhanced. These states are supported by the Efimov potential which scales like $-1 / R^{2}$ for $R<|a|$, where the hyperspherical radius $R$ characterizes the geometric size of the system. ${ }^{6}$

The connection between the three-body Efimov potential and scattering length $a$ can be understood using a hand-waving picture. Assume two bosonic atoms are separated by $R$, the wave function of the third atom is scattered by each atom, with $|a|$ characterizing the length scale of the scattered waves. The total wave function $\psi$, after Bose-symmetrization, can be significantly enhanced when the two scattered waves overlap. Using Schrödinger's equation, we can model the wave function enhancement $\phi(R)=\delta \psi(R)$ as a result of an effective Efimov potential $V_{\text {efm }}(R)$, which satisfies Schrodinger's equation:

$$
-\frac{\hbar^{2}}{m} \phi^{\prime \prime}(R)+V_{\text {efm }}(R) \phi(R)=E \phi(R) \quad \text { for } R<a .
$$

To evaluate the curvature term, we note that $\phi$ is localized with a length scale of system size $R$. The curvature is thus negative and we can rewrite $\phi^{\prime \prime}(R)=-\alpha \phi(R) / R^{2}$, where $\alpha>0$ is a proportionality constant. In the low energy collision limit $E \rightarrow 0$, we get

$$
V_{\mathrm{efm}}(R)=-\frac{\alpha \hbar^{2}}{m R^{2}} \quad \text { for } R<a,
$$


A rigorous calculation performed by V. N. Efimov shows that with $R$ identified as the three-body hyperspherical radius, we have $\alpha=s_{0}^{2}+\frac{1}{4}$ and $s_{0}=1.00624 \ldots$ is a constant. ${ }^{6}$ For $R>|a|$, the effective potential is no longer attractive. ${ }^{14}$

Right on two-body resonance $a \rightarrow \pm \infty$, the $-1 / R^{2}$ Efimov potential extends to infinity and can support an infinite number of three-body bound states (Efimov states); simple scaling laws for the spatial extent $A_{N}$ and binding energy $E_{N}$ of the $N$-th lowest Efimov state have been derived as

$$
\begin{aligned}
& A_{N}=\beta^{N} \times A^{*} \\
& E_{N}=\beta^{-2 N} \times E^{*},
\end{aligned}
$$

where $\beta=e^{\pi / s_{0}} \approx 22.7$ is a universal constant. ${ }^{6}$ These size and energy scaling laws are among the most prominent universal features of Efimov's predictions. Constants $A^{*}$ and $E^{*}$ depend on the three-body potential at short range and are thus expected to be non-universal. ${ }^{2}$

\subsection{Universality of Efimov physics near different Feshbach resonances}

Recent observation of an Efimov resonance in the three-body recombination process $^{15}$ of ultracold cesium atoms represents a major breakthrough in few-body physics. ${ }^{16}$

Here we suggest a new scheme to check the "defacto" universality of Efimov physics implied by the expected slow variation of the short-range three-body potential with magnetic field tuning. By monitoring recombination loss near different, isolated open-channel dominated Feshbach resonances, we expect that Efimov resonances of the same order can occur at the same scattering lengths. Here we point out that the application of magnetic field barely changes the interatomic potential in the entrance scattering channel. We thus expect that, in the three-body sector, systems have nearly identical off-resonant phase shift near different Feshbach resonances.

To estimate the insensitivity of the open channel potential to magnetic field, we note that the two-body background scattering length varies less than $1 a_{0}$ over $100 \mathrm{G}$ at $a=2400 a_{0}$. (This estimation is based on numerical calculation of cesium atom scattering length in the highest triplet scattering channel, in which Feshbach resonances do not exist.) This small variation can be translated into a small fractional change of the scattering phase shift by $|\delta \eta / \eta|<3 \times 10^{-10}$ per Gauss. ${ }^{17}$ This result suggests that the non-universal effects of the three-body potential can potentially remain 
nearly unchanged when the magnetic field is tuned to different Feshbach resonances.

\section{Universality in a dilute BEC with large scattering length: Lee-Huang-Yang Corrections and beyond}

In a dilute Bose-Einstein condensate, the energy per particle is given as $2 \pi n a \hbar^{2} / m$, which describes the fluid on length scales longer than the coherence length $l=(16 n a)^{-1 / 2}$. Due to the weak coupling, corrections to the mean field term can be calculated as expansions of a dimensionless parameter $a / l$, which is in turn proportional to the diluteness parameter $\sqrt{n a^{3}}$. The energy per particle in a dilute homogeneous BEC is given by ${ }^{20}$

$$
\frac{E}{N}=\frac{2 \pi \hbar^{2} n a}{m}\left[1+\frac{128}{15 \sqrt{\pi}} \sqrt{n a^{3}}+\frac{8(4 \pi-3 \sqrt{3})}{3} n a^{3} \ln n a^{3}+C n a^{3}+\ldots\right],
$$

where the lowest order contributions $\sqrt{n a^{3}}$, called the Lee-Huang-Yang (LHY) correction, ${ }^{18}$ and $n a^{3} \ln n a^{3}$ term ${ }^{19}$ result from universal two- and three-body correlations, and $C$ is a three-body parameter which depends on three-body interactions and Efimov physics. ${ }^{20}$ Although Eq. 8 was originally derived based on a hard-sphere potential, the validity of the LHY term for soft-sphere and short-ranged attractive potentials has been numerically verified..$^{21}$

Beyond mean-field effects can be amplified by tuning the scattering length to large values. Previous approaches along this line with ${ }^{85} \mathrm{Rb}$ reached $n a^{3}=0.1$, but were complicated by limited lifetimes due to threebody inelastic collisions. Here we point out that a careful choice of scattering length and a fast measurement can allow for a detectable beyond mean-field signal.

To see this, we first note that the LHY term, on the order of $\left(n a^{3}\right)^{1 / 2}$, is a lower order process than is the three body process of $n a^{3}$. Measurement of the former effect can be immune from three-body loss when $n a^{3}$ is low. For example, the typical mean-field energy of a BEC is $U=\mathrm{h} \times 1 \mathrm{kHz}$ and the scattering length can be tuned such that $n a^{3}=0.01$. In this case, the LHY term is about $\left(n a^{3}\right)^{1 / 2}=10 \%$ of the mean-field energy and is 10 times larger than the three-body energy scale. The associated three-body time scale is $n a^{3} U / \hbar \approx(10 \mathrm{~ms})^{-1}$. Determination of interaction energy of a condensate within $10 \mathrm{~ms}$ can be realized by promptly releasing the condensate into free space. The expansion of the condensate thus converts the interaction energy into detectable atomic kinetic energy. 


\section{Experimental approach}

Two powerful experimental tools will be employed to explore few-body physics: optical lattices to confine and isolate few atoms at each lattice site in the Mott insulator phase, and magnetic Feshbach resonances to control atomic interactions. Both can lead to precise control of the fewbody samples in different interaction regimes.

\subsection{Scattering properties of cesium atoms}

Cesium-133 is chosen in the experiment for their convenient tuning of interaction. In the range of 0 to $50 \mathrm{G}$, the s-wave scattering length in the lowest hyperfine ground state $\left|F=3, m_{F}=3\right\rangle$ can be smoothly tuned from $-2500 a_{0}$ to $1000 a_{0}$. Here, $F$ is the total angular momentum quantum number and $m_{F}$ its projection along the magnetic field. At higher fields, two more broad resonances exist at $547 \mathrm{G}$ and 800 G. See Fig. 2.

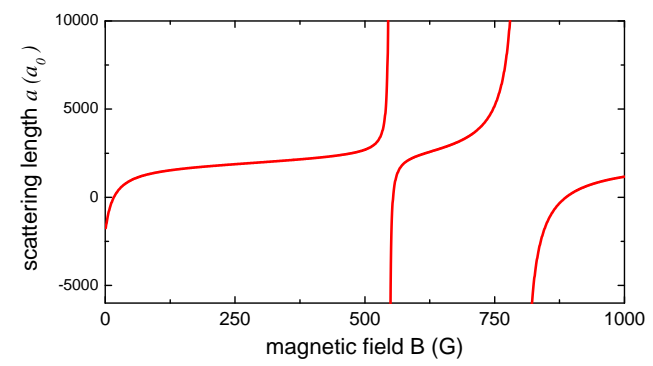

Fig. 2. Calculated $s$-wave Feshbach resonances in collisions of ground state cesium atoms. Three broad resonances at $-11.7 \mathrm{G}, 547 \mathrm{G}$ and $800 \mathrm{G}$ allow for tuning of the scattering length. Other higher-order resonances are omitted here for simplicity. The numerical calculation code is provided by Eite Tiesinga, NIST.

The existence of multiple broad $s$-wave Feshbach resonances permits tests of universality by probing the cold atoms sample at different scattering lengths. As discussed in Secs. 3.2 and 4.1, unique tests of universality in two- and three-body systems can be performed by tuning the scattering length to the same value, but near different Feshbach resonances.

\subsection{Fast evaporation to Bose-Einstein condensation in optical traps}

We employ a novel scheme to achieve fast, runaway evaporative cooling of cesium atoms in optical traps. This is realized by tilting the optical potential 
with a magnetic field gradient. Runaway evaporation is possible in this trap geometry due to the very weak dependence of vibration frequencies on trap depth, which preserves atomic density during the evaporation process. When the trap depth is reduced by a large factor of 100 , the geometric mean of the trap frequencies is only reduced by a factor of 2 and thus preserves the high collision rate. ${ }^{22}$

Using this scheme, we show that Bose-Einstein condensation with $10^{5}$ cesium atoms can be realized in $2 \sim 4$ s of forced evaporation. ${ }^{22}$ The evaporation speed and energetics are consistent with the three-dimensional evaporation picture, despite the fact that atoms can only leave the trap in the direction of tilt.

\subsection{Preparation of few-atom systems in optical lattices}

Few-body experiments will begin with segmentation of a bulk condensed superfluid into the ground states of isolated optical lattice sites. Each site will be populated with a small and in general indefinite number, $1<N<$ 10 , of atoms.
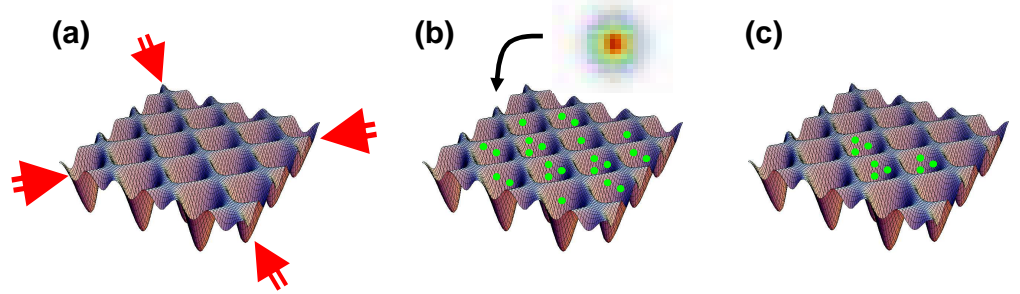

Fig. 3. Loading an optical lattice and preparation of lattice sites with three atoms. (a) Optical lattices are formed by the interference pattern of intersecting laser beams. For a red-detuned lattice, the potential minima are defined by the anti-nodes of the standing waves. (b) Condensed atoms are loaded into the optical lattices. (c) To prepare lattice sites with three and only three atoms, atoms in lattice sites with other occupancies may be removed by precision radio-frequency excitation.

We have constructed a novel optical lattice configuration - a thin layer, 2D optical lattice, which permits direct imaging of atomic density by sending an imaging beam perpendicular to the lattice plane. The optical lattice is defined by interfering four laser beams derived from a single frequency fiber laser operated at a wavelength $\lambda=1.06 \mu \mathrm{m}$. Two counterpropagating beam pairs on the horizontal plane form a square optical lattice. In the ver- 
tical direction, confinement is provided by a single $\mathrm{CO}_{2}$ laser beam focused to $50 \mu \mathrm{m}$ vertically and $2 \mathrm{~mm}$ in the radial direction. This tight vertical confinement holds atoms against gravity without need for a magnetic field gradient, and provides an ideal mode-matching potential for transferring condensates into the 2D lattice, see Fig. 3.

Probing of few-body energies will be performed through the combined methods of precision radio-frequency spectroscopy, collective mode excitation and dynamic evolution of matter wave coherence. In particular, methods which allow precise determination of the variation of few-body energies with atom number will permit direct investigation of various interactions, including two- and three-body scattering of free atoms, strong correlations, unitarity at strong confinement, atom-dimer interactions, and three-body recombination. Working in a tightly bound optical lattice allows quantum pressure to determine atomic density profiles and permits accurate extrapolation of single-particle measurements to interacting few-body systems. In addition, methods of adiabatically preparing specific few-body systems as combinations of free and bound states (e.g. atom+dimer) will be explored, providing a basis for directly studying the universality of higher complexity interactions.

\section{Conclusion}

We describe key issues in three- and many-body physics including Efimov physics and beyond mean-field effects in the context of Feshbach tuning in quantum gases. In both cases, Bose-condensed cesium atoms provide unique opportunities to investigate universal behavior of energy shifts and energy structure. In particular, we point out possible non-universal parameters, including the finite interaction range $\bar{a}$, Feshbach coupling length scale $R^{*}$ and three-body phase shift.

We propose a brand new approach to prepare and study few-body systems in optical lattices by inducing superfluid-Mott insulator transition in a single layer of 2D optical lattices. This system provides complete and independent control over the filling factor, on site interaction and tunneling. Together with the rich interaction properties of cesium atoms and fast evaporation, one expect new level of few-body physics can be explored in this lattice setting. We anticipate that a firm understanding of universality in finite systems will provide practical applications in quantum simulation of few-body systems in nuclear physics, helium physics, physical chemistry and the physics of atom clusters. 


\section{Acknowledgement}

The authors acknowledge support from the NSF-MRSEC program under No. DMR-0213745, ARO Grant No. W911NF0710576 from the DARPA OLE program and Packard foundation. N.G. acknowledges support from the Grainger Foundation.

\section{References}

1. S. Inouye, M. R. Andrews, J. Stenger, H.-J. Miesner and D. M. Stamper-Kurn and W. Ketterle, Nature 392, 151 (1998).

2. E. Braaten and H.-W. Hammer,Phys. Rep. 428, 259 (2006).

3. C. A. Regal, M. Greiner and D. S. Jin, Phys. Rev. Lett. 92, 040403 (2004).

4. M. Bartenstein, A. Altmeyer, S. Riedl, S. Jochim, C. Chin, J. Hecker Denschlag and R. Grimm, Phys. Rev. Lett. 92, 120401 (2004).

5. M. W. Zwierlein, C. A. Stan, C. H. Schunck, S. M. F. Raupach, A. J. Kerman and W. Ketterle, Phys. Rev. Lett. 92, 120403 (2004).

6. V. Efimov, Phys. Lett. B 33, 563 (1970).

7. E. Braaten, H.-W. Hammer, and T. S. Mehen, Phys. Rev. Lett. 88, 040401 (2002).

8. E. Tiesinga, B.J. Verhaar, B. J. and H.T.C. Stoof, Phys. Rev. A, 47, 4114 (1993).

9. N. F. Mott and H. D. W. Massey, "Theory of atomic collisions", Oxford University Press, London, (1965).

10. P. S. Julienne and B. Gao, Atomic Physics 20, Edt. by C. Roos and H. Häffner and R. Blatt, AIP, Melville, New York, pp. 261-268 (2006).

11. G. F. Gribakin and V. V. Flambaum, Phys. Rev. A 48, 546 (1993).

12. C. Chin, R. Grimm, E. Tiesinga and P.S. Julienne, (to be submitted to Rev. Mod. Phys.)

13. D. S. Petrov, Phys. Rev. Lett. 93, 143201 (2004).

14. J. P. D'Incao and B. D. Esry, Phys. Rev. Lett. 94, 213201 (2005).

15. B. D. Esry, C. H. Greene and J. P. Burke,Phys. Rev. Lett. 83, 1751 (1999).

16. T. Kraemer, M. Mark, P. Waldburger, J. G. Danzl, C. Chin, B. Engeser, A.D. Lange, K. Pilch, A. Jaakkola, H.-C. Nägerl and R. Grimm, Nature 440, 315 (2006).

17. C. Chin and V. V. Flambaum, Phys. Rev. Lett. 96, 230801 (2006).

18. T. D. Lee, K. Huang, and C. N. Yang, Phys. Rev. 106, 1135 (1957).

19. T. T. Wu, Phys. Rev. 115, 1390 (1959).

20. E. Braaten, H.-W. Hammer, and T.S. Mehen, Phys. Rev. Lett. 88, 040401 (2002).

21. S. Giorgini, J. Boronat, and J. Casulleras, Phys. Rev. A 60, 5129 (1999).

22. C.-L. Hung, X. Zhang, N. Gemelke, and C. Chin, Phys. Rev. A 78, 011604 (2008). 